\title{
Vectorial dissipative solitons in vertical-cavity surface-emitting lasers with delays
}

\author{
M. Marconi, ${ }^{1}$ J. Javaloyes, ${ }^{2}$ S. Barland,${ }^{1}$ S. Balle, ${ }^{3}$ and M. Giudici ${ }^{1}$ \\ ${ }^{1}$ Institut Non Linéaire de Nice, Université de Nice Sophia Antipolis - Centre National de la Recherche Scientifique, \\ 1361 route des lucioles, F-06560 Valbonne, France \\ ${ }^{2}$ Departament de Fisica, Universitat de les Illes Baleares, \\ C/ Valldemossa, $\mathrm{km}$ 7.5, E-07122 Palma de Mallorca \\ ${ }^{3}$ Institut Mediterrani d'Estudis Avançats, CSIC-UIB, E-07071 Palma de Mallorca, Spain
}

\begin{abstract}
We show that the nonlinear polarization dynamics of a vertical-cavity surface-emitting laser placed into an external cavity leads to the formation of temporal vectorial dissipative solitons. These solitons arise as cycles in the polarization orientation, leaving the total intensity constant. When the cavity round-trip is much longer than their duration, several independent solitons as well as bound states (molecules) may be hosted in the cavity. All these solutions coexist together and with the background solution, i.e. the solution with zero soliton. The theoretical proof of localization is given by the analysis of the Floquet exponents. Finally, we reduce the dynamics to a single delayed equation for the polarization orientation allowing interpreting the vectorial solitons as polarization kinks.
\end{abstract}

\section{INTRODUCTION}

The field of dissipative solitons (DS) in optical systems has been the subject of an intensive research during the last twenty years (see e.g. 115] and references therein). Optical DS are localized light pulses in time or localized beams in space appearing in nonlinear systems kept out of equilibrium by a continuous flow of energy counteracting the losses. As a consequence, they exhibit remarkable differences with respect to their conservative counterparts which arise purely as the result of a nonlinearity compensating for the spreading effects.

DS are attractors, i.e. stable solutions towards which the system evolves spontaneously from a wide set of initial conditions [6. It entails that, at variance with their conservative analogues, DS do not rely on a proper seeding of the initial conditions. This renders them extremely interesting for applications and, in particular, for information processing. As they coexist with the background solution, they can be individually addressed and used as information bits [7]. All-optical buffers were demonstrated using both spatial and temporal DS [3, 8, 11].

Another important aspect concerns the role of the nonlinearity. While in the beginning DS were envisioned as weakly modified conservative solitons [12, it was latter shown that for strong dissipation, the role of the nonlinearity leading to their formation is not limited to the compensation of the spreading effect. Dissipative Solitons are known to occur both in the normal and the abnormal dispersion regimes [13]. For instance, the socalled Cavity Solitons appearing in the transverse plane of driven resonators [8, 9, 14, 17] have been described as localized structures, i.e. as elementary cellular patterns (or cellular pulses) generated by fronts connecting different coexisting spatial solutions [18]. In general, CS may appear even in presence of defocussing nonlinearities, i.e. which favor the spatio-temporal spreading effect [17].

Temporal DS have been largely studied in long-cavity mode-locked fiber lasers [5], and several interesting behaviors have been experimentally demonstrated, as e.g. soliton bound states [19, molecules 20, 21, repulsive/attracting forces on extremely long scale [22, 23], soliton rain 24] or soliton explosion [25]. When the vectorial degree of freedom of the light is taken into account like e.g. in the case of the Manakov solitons [26, more complex behaviors are observed. Anti-phase switching between orthogonally linearly polarized states has been recently observed [27, 28] and interpreted in terms of soliton domain walls, i.e. localized states separating domains of emission in orthogonal polarizations [28, 29].

In this manuscript we report evidence of temporal vectorial DS in a single mode Vertical-Cavity SurfaceEmitting Laser (VCSEL) enclosed into a polarization sensitive double external cavity. By exploiting the two different times of flight as well as the polarization selectivity of the cavity, we are able to control the polarization state of light and generate vectorial DS. They consist in time-localized rotations of the emitted polarization orientation, thus leaving the total intensity constant. When the cavity round-trip is much longer than the duration of the vectorial DS, several independent DS and/or DS bound states (molecules) may be hosted in the cavity where they coexist with the background solution (zero DS emission). We show that molecules and independent DS can be discriminated experimentally by analyzing their noise-induced motion.

The vectorial nature of the reported DS is intimately related to the nonlinear polarization response of the VCSEL. Because of their axial symmetry, these devices lack strong enough anisotropies to pin the polarization orientation [30 32 and competition between orthogonal linearly polarized states is easily induced by polarized perturbations. Such dynamics doesn't involve a strong exchange of energy between the light and the active medium, and the addressing speed of these DS shall not be limited by the nanosecond timescale governing the evolution of the gain. While the complex polarization dynamics of VCSEL was recently exploited to generate chaotic states for data encryption [33], we show in this manuscript that it can also be harnessed for all-optical in- 
formation encoding where bits of information are stored in the form of vectorial DS as short as $25 \mathrm{ps}$, as predicted by our analysis.

From the theoretical point of view, it is important to point out that the nonlinearity in our system is concentrated on a single point (the VCSEL) rather than being distributed along the propagation direction as in fiber resonators. For this reason, we model our DS by Delay Differential Equations (DDEs) rather than by Partial Differential Equations (PDEs), as the prototypic cubicquintic Ginzburg-Landau equation of DS. In fact DDEs possess the same complexity as PDEs since they both correspond to dynamical systems of infinite dimensionality. Moreover, conceptual links between PDEs and DDEs do exist. It was revealed that a delayed system close to an Andronov-Hopf bifurcation can be described via a Ginzburg-Landau equation [34] and recently, a mapping between a spatially extended laser cavity and an ensemble of coupled Delay Algebraic Equation was developed [35, 36]. In this manuscript, we establish a criterion for temporal localization in DDE systems based upon the degeneracy of the Floquet exponents. Finally, we reduce the full model to a single delayed equation for the polarization orientation allowing interpreting the vectorial solitons as polarization kinks and anti-kinks.

\section{EXPERIMENTAL RESULTS}

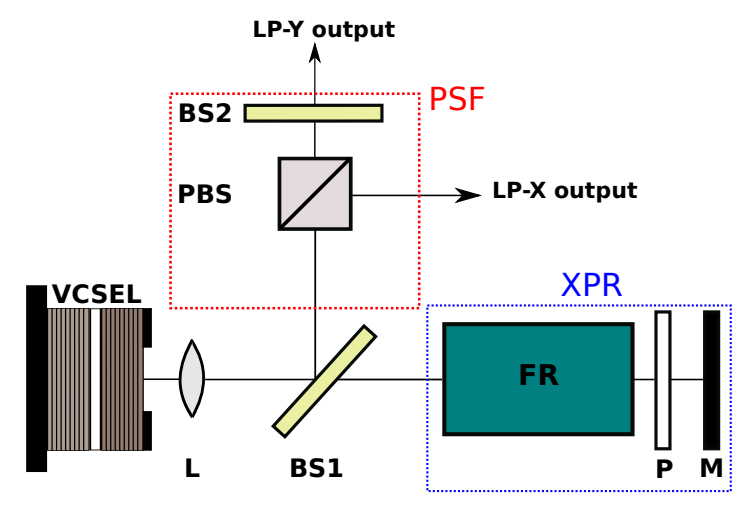

Figure 1. Experimental set-up. The light emitted is collimated by an aspheric lens (L) and split in two beams by a polarization preserving beam-splitter (BS1). In the PSF branch, a polarizing cube (PBS) transmits only the Y component towards a partially reflective mirror (BS2) that feeds back $\mathrm{Y}$ into the VCSEL. The PBS sends the X component out of the cavity for detection while the detection of the $\mathrm{Y}$ component is obtained using the beam transmitted by BS2. In the XPR branch, the beam is sent to a $\pi / 4$ Faraday rotator $(\mathrm{FR})$ with an exit polarizer $(\mathrm{P})$ transmitting only the $\mathrm{Y}$ component that will be rotated into $\mathrm{X}$. Then, the mirror $\mathrm{M}$ reflects the light back towards the VCSEL. Because of the double passage into the $\mathrm{FR}, \mathrm{Y}$ is finally re-injected into the VCSEL with a polarization axis parallel to X. Neutral density filters in both branches allow varying feedback levels.

The experimental setup is schematically shown in
Fig. 1 and more details can be found in Methods IV A. A single-transverse mode VCSEL is coupled to a double external cavity that selects one of the linearly polarized states of the VCSEL (Y, say) and feeds it back twice into the VCSEL: once into the same polarization and once into the orthogonal one. The first arm provides Polarization Selective Feedback (PSF) with a time delay $\tau_{f}$, while the second arm re-injects Y into the orthogonal polarization orientation with time delay $\tau_{r}$. Such CrossedPolarization Re-injection (XPR) induces cross-gain modulation of the two linear polarization components [32], thus enhancing their competition and leading to polarization dynamics 37, 38. This double cavity was shown to promote Passive-Mode-Locking without saturable absorber 39, though here it is operated in a completely different regime.

For properly chosen parameters (see Methods IV A), the polarization resolved outputs of the VCSEL exhibit a train of pulses separated by $\tau_{f}=5.6 \mathrm{~ns}$, as shown in Fig. 2a. In the $\mathrm{X}$ polarization component, the pulses are upward over a low intensity background while they are downward from a high intensity level in Y. The pulse duration is $80 \mathrm{ps}$ (FWHM) and it is not fully resolved due to the bandwidth limitation of the detector used ( 8 $\mathrm{GHz}$ ). These polarization pulses appear anti-correlated and the corresponding total intensity time trace is almost constant, thus revealing the vectorial character of this dynamics. We interpret these polarization pulses as vectorial DS which travel back and forth in the external cavity and get regenerated at each round-trip when interacting with the VCSEL.

Dissipative solitons are required to maintain their shape throughout the number of round-trips covered, though inevitable noise sources (namely spontaneous emission in the laser, detector shot noise, mechanical and thermal stability of the experimental set-up) can blur this ideal picture, as shown in 9. We assessed the self-similarity of the pulses by performing a statistical analysis of an $100 \mu$ s long time series spanning over $18 \times 10^{3}$ pulses. The distribution of the maxima of the pulses exhibits a Gaussian shape with a standard deviation of $\sim 10 \%$ of the average peak intensity. By using the peak of each pulse as a time reference, we superposed the $18 \times 10^{3}$ waveforms in Fig. $2 \mathrm{p}$. This reveals that, regardless of the peak intensity fluctuations, the shape of the pulse remains robust and stable, thus supporting our interpretation of the pulses in terms of vectorial DS.

The dynamics within an external cavity can be usefully described in terms of space-time like diagrams where the time trace is folded over itself at intervals $\tau_{f}$, so that the round-trip number becomes the pseudo-time discrete variable while the pseudo-space variable corresponds to the timing of the vectorial DS modulo $\tau_{f}$ 34, 40. This representation pictures the soliton position $\Delta_{n}$ within the external cavity as a function of the round-trip number [23. When applied to the $\mathrm{X}$ polarization trace of Fig. 2 a it reveals that $\Delta_{n}$ fluctuates noticeably over a typical time scale of the order of a hundred of cycles, suggesting 

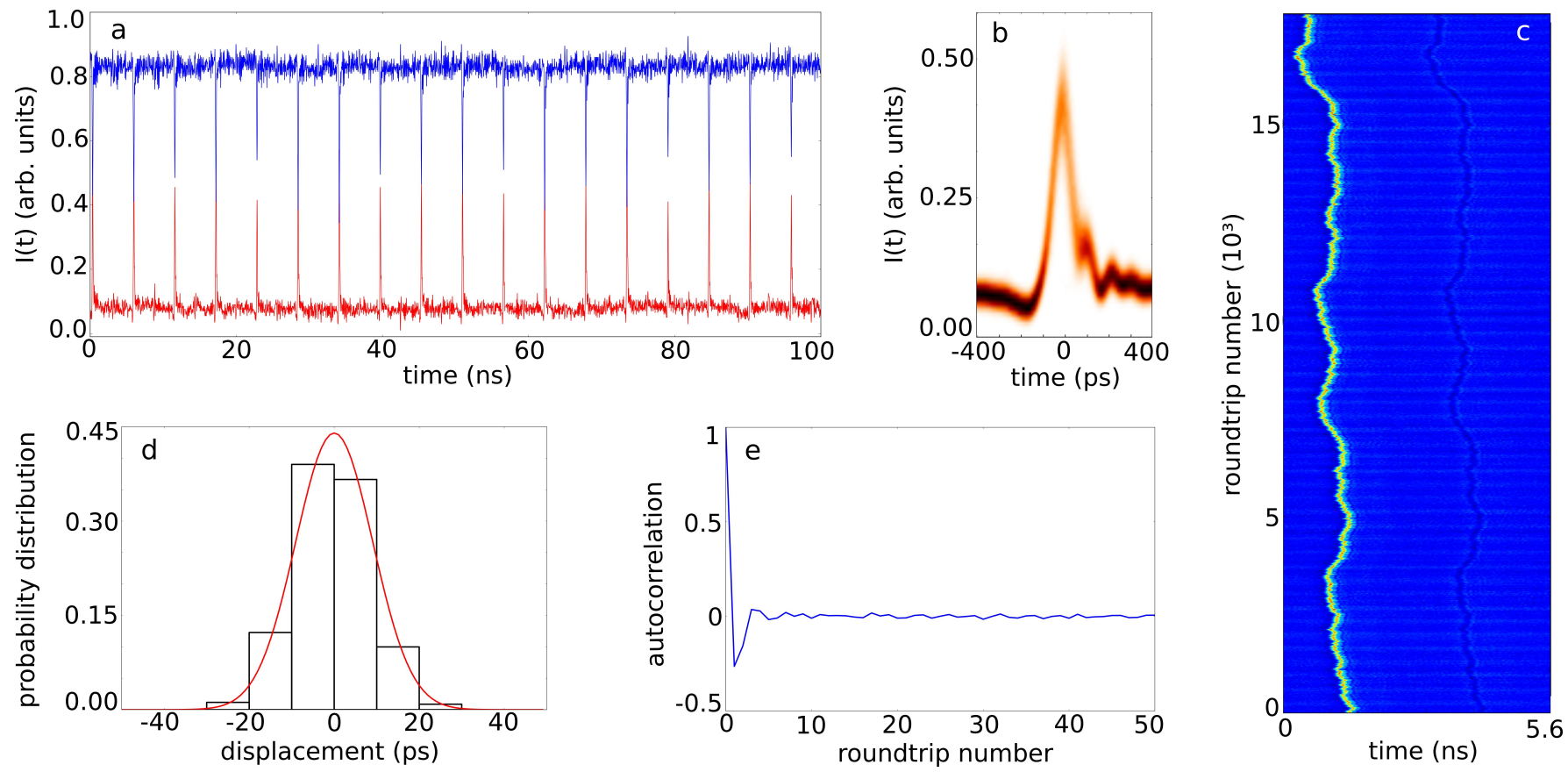

Figure 2. Single Dissipative Soliton regime. Panel a): Time signal of the Y (blue) and X (red) outputs when the VCSEL is submitted to XPR (rate $1.4 \%$ ) and PSF (rate $0.6 \%$ ). $J=3.0 \mathrm{~mA}, \tau_{f}=5.6 \mathrm{~ns}, \tau_{r}=8.4 \mathrm{~ns}$. The double trace acquisition limits the sampling rate at $100 \mathrm{GS} / \mathrm{s}$ Panel b): Histogram in color grade persistence resulting from the superposition of $18 \times 10^{3}$ pulses in the X polarization obtained using the same time trace as in b). Panel c): Space-time representation diagram of a single DS obtained using the same time trace as in b). The shadow to the right of the pulse is almost invisible in the time trace and corresponds to a small inverted pulse (i.e. upward in $\mathrm{Y}$ and downward in X) that appears at time $\Delta \tau=\tau_{r}-\tau_{f}=2.8 \mathrm{~ns}$. Panel d): Probability distribution of the residual $R_{n}=\Delta_{n}-\Delta_{n-1}$ calculated from the trace in a) with $\Delta_{n}$ the position of the pulse at round-trip $n$ in c). Panel e) Autocorrelation of $R_{n}$.

that the noise present in the system acts upon the soliton as a Langevin force over a free particle, leading to a Brownian-like motion of the soliton within the external cavity as a function of the round-trips covered. Indeed, the analysis of the time series for $\Delta_{n}$ indicates that this variable is described by a first order autoregressive model, $\Delta_{n}=\Delta_{n-1}+R_{n}$, with $R_{n}$ a random term which is distributed as a Gaussian of zero mean and a standard deviation $\sigma_{R}=11 \mathrm{ps}$, as shown in Fig. 2 $\mathrm{d}$. Moreover, the autocorrelation of $R_{n}$ (Fig. 2p) falls-off very rapidly (a few round-trips) indicating that the sequence of $\Delta_{n}$ corresponds to the regular sampling of an one-dimensional stochastic process $d_{t} \Delta=\xi(t)$ where $\xi(t)$ is a Gaussian, slightly colored noise. The analogy between unidimensional Brownian motion of a free particle and the drift of the DS position within the cavity can be traced back to the temporal translational invariance of our autonomous dynamical system [41, 42].

If $\tau_{f}$ is large enough compared with the size of a DS, several localized structures can be hosted within the external cavity and their interaction may be studied. Then, the noise induced motion of the DS becomes a tool for discriminating between independent DS and DS bound states or molecules. We show in Fig. 3a-c the space-time diagrams for different ensembles of coexisting DS. We remark that these different realizations have been obtained for the same parameter values; the system may evolve from one situation to another in response to perturbations or parameter sweeps, in the latter case displaying a high degree of hysteresis. Panel a) shows two DS whose noise-induced trajectories are uncorrelated, thus evidencing their independence. Panel b) shows a molecule of three DS; while the evolution of the ensemble is stochastic, the separation between the DS remains constant at $480 \mathrm{ps}$, which corresponds to $\Delta \tau=\tau_{r}-\tau_{f}$ (Supplementary Section VA. Fig. 8a,b). We depict in Fig. 3p the binding and unbinding between two molecules, each one formed by two DS. At round-trip $12 \times 10^{3}$, the two molecules approach and form a bound state with four peaks that afterwards moves as a single structure and subsequently unbind and bind again at latter times. The situations reported in Fig. $3 \mathrm{a}-\mathrm{c}$ are a small sample of the multiple situations in terms of DS number and organization (molecules of variable number of DS) that coexist for the same values of the parameters. For $\tau_{f}=2.9 \mathrm{~ns}$, molecules composed by a larger number of DS can be observed (Supplementary Section VA Fig. 7a), the largest one being an 8-DS molecule filling the entire round-trip and forming a DS "crystal". Increasing the size of the external cavity allows placing a higher number of independent DS and larger molecules (Supplementary Section $\mathrm{VA}$. Fig. 7b,c). In Fig. 3d we show how different 


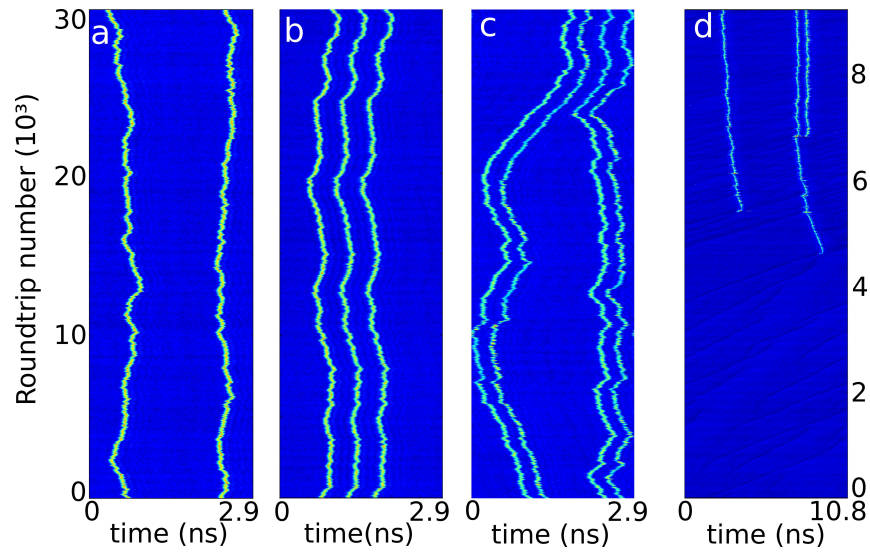

Figure 3. Space-time like diagrams of different states with multiple solitons coexisting within the external-cavity. a) Two independent DS, b) a 3-DS molecule separated by a time $\Delta \tau$, c) two independent 2-DS molecules that form a 4-DS molecule at round-trip $12 \times 10^{3}$ separate and bind again at round-trip $23 \times 10^{3}$. In $(\mathrm{a}-\mathrm{c}) \tau_{f}=2.9 \mathrm{~ns}$ and $\tau_{r}=3.3 \mathrm{~ns}$. In d) we represent a similar dynamics yet for longer delays $\tau_{f}=10.8 \mathrm{~ns}$ and $\tau_{r}=11.13 \mathrm{~ns}$; here several vectorial DS spontaneously nucleate due to noise induced fluctuations at round-trip $4.5 \times$ $10^{3}, 5.2 \times 10^{3}$ and $7 \times 10^{3}$, thus evidencing the multi-stability with the CW solution. While the first and second DS are independent, the third one forms a molecule with the second at a bonding distance of $600 \mathrm{ps}$, i.e. at a distance twice the nominal separation, i.e. $2 \Delta \tau$. All the other parameters are as in Fig. 2

vectorial DS may nucleate spontaneously from the CW solution, i.e. from a situation with zero solitons. Fig. 3d suggests that DS can be addressed inside the cavity by a proper perturbation pulse and used as bits for information processing, as reported in [9].

\section{THEORY}

In order to explain the experimental results, we use the Spin-Flip Model [43, suitably modified for incorporating the effects of gain saturation, PSF and XPR. The choice of parameter values was guided by the experimental situation (see Methods IV B). Fig. 4 a shows a dynamical state for the 1-DS case in good agreement with the experiment: the intensity of each linearly polarized component displays localized anti-phase pulsations separated by the PSF time delay, followed by a small inverted kink after a time $\Delta \tau$, see Fig. $4 \mathrm{~b}$. The presence of this inverted kink in the experimental data can be appreciated in Fig. 25 as a dark shadow following the DS at a distance $\Delta \tau$. We predict an almost $100 \%$ anti-phase as well as pulse-widths of the order of $\sim 35$ ps which is compatible with the experimental results taking into account the bandwidth limitations. Importantly, such regime coexists with a CW solution and in-between pulses (or in the absence of them), the emission consists in a quasi-linearly polarized mode whose orientation is neither $\mathrm{X}$ nor $\mathrm{Y}$. The
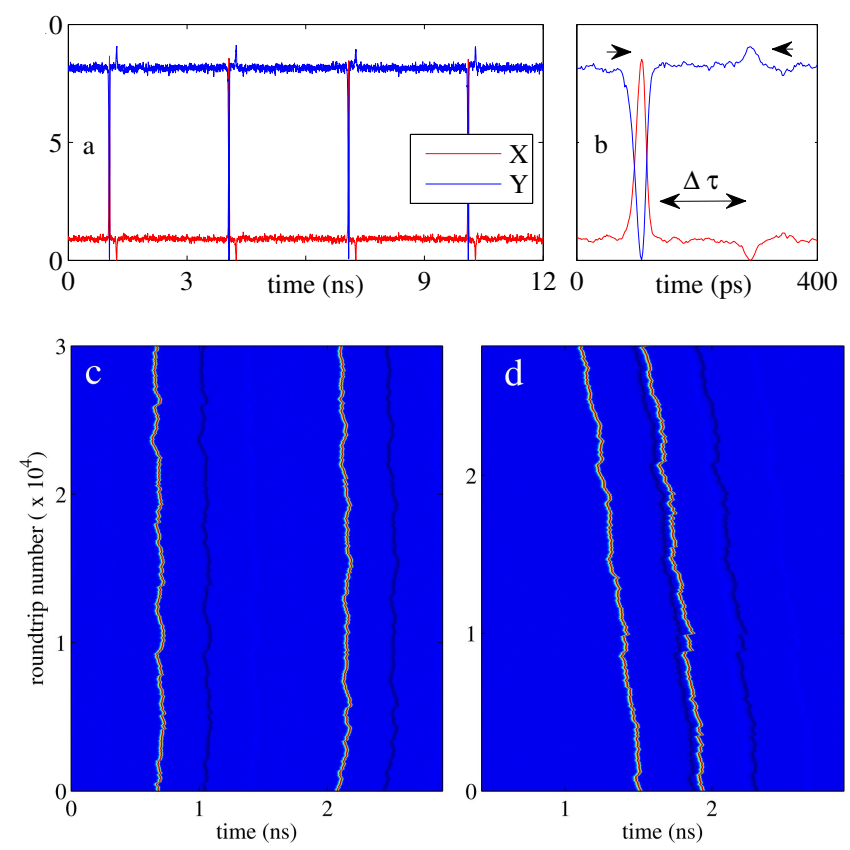

Figure 4. Theoretical temporal trace for a single vectorial DS (a) created by perturbing the phase of PSF (see Methods IV B and (b) zoom on the pulse detail: the anti-phase dip is followed by a small inverted kink after a time delay $\Delta \tau$ corresponding to the re-injection of $\mathrm{Y}$ into $\mathrm{X}$ after a longer time $\tau_{r}$. Other exponentially decreasing replica of this secondary kink (not visible) follow at time intervals $n \Delta \tau$. (c-d) Folded space-time representation: the shadow following the DS corresponds to the inverted dip at $\Delta \tau$. In (c) the second DS was created far from the first one yielding two independent objects as evidenced by their uncorrelated motion. Conversely, if the second DS is nucleated at some precise closer distance, one obtains a bound state (d) where the two solitons exhibit correlated motions. The period of the solutions is slightly superior to $\tau_{f}$, such secular drift being due to the finite response time of the VCSEL.

polarization orientation is governed by a complex interplay between the dichroism, the birefringence, the PSF and the XPR rates as well as the two delays, see 38, for details. Typically, the suppression ratio between $I_{y} / I_{x}$ can be tuned between 20 and 5 .

In addition to isolated DS, we reproduce the coexistence of multiple independent solitons, see Fig. 4. Additional DS can be written at arbitrary positions without perturbing the already present localized structures. The bound states are accounted for as well, which can be appreciated in Fig. 4 d. We explain the existence of a specific binding distance to the small kink generated in the wake of the vectorial DS by the replica of the main DS impinging the VCSEL a second time after a time interval $\Delta \tau$, in agreement to experimental results of Fig. 2 c. Notice that several exponentially decreasing replica at time $2 \Delta \tau, 3 \Delta \tau$ exist and generate a weak binding force as well as other equilibrium distances for the molecules (Supplementary Section VD. Finally, for slightly different 
parameters values, a more complicated situation exists in which the background $\mathrm{CW}$ solution weakly oscillates in anti-phase for $\mathrm{X}$ and $\mathrm{Y}$ at a frequency close to the birefringence. This induces additional binding distances whose separation is $\sim 120 \mathrm{ps}$ around the main resonances $n \Delta \tau$. Such complexity is typical of the interaction between vectorial DS, see e.g. [44, and will be reported elsewhere.

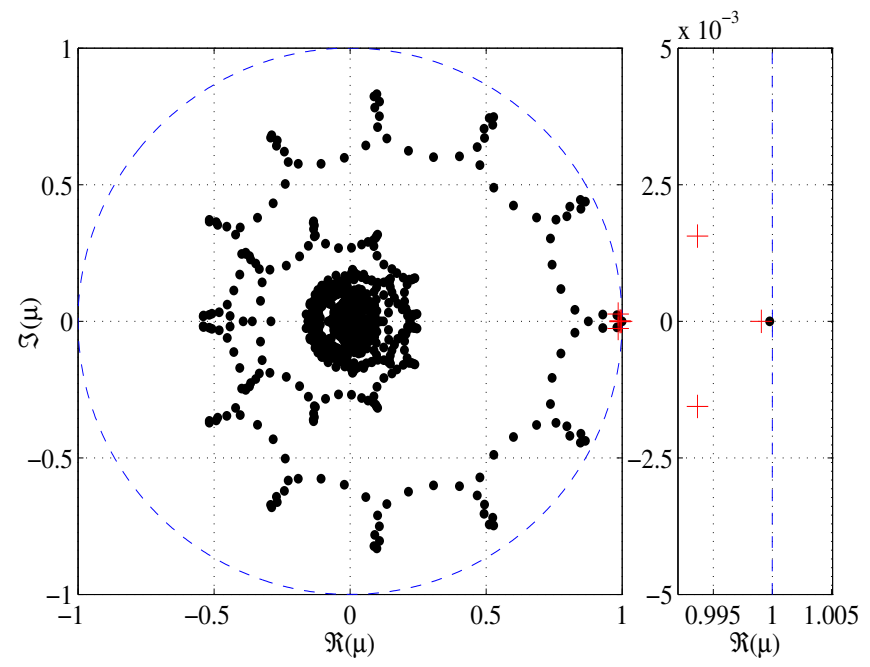

Figure 5. Floquet multipliers $\mu$ (black circles) of the single DS solution showed in Fig. 4 4 . All the multipliers have a modulus smaller than unity, as expected for a stable solution. A zoom in the vicinity of $\mu=1$ allows finding a single multiplier as anticipated for a periodic solution in a time independent dynamical system. For the case of $N=3 \mathrm{DS}$, we represent for clarity only the rightmost multipliers (red crosses): we notice the existence of not one but three quasi-degenerate Floquet multipliers having no equivalent in the case of the single DS. This demonstrates the existence of $N$ degenerate neutral mode that correspond to independent translational motion of the various DS. The fact that the three multipliers are not exactly equal to unity is a consequence of the ultraweak residual interactions between distant DS. Such residual interaction implies that — strictly speaking — multiple DS are not totally independent for any finite value of the delay.

As a proof of the independence of the DS, we performed the analysis of the Floquet multipliers considering the DS as periodic solutions of a high dimensional dynamical system (Supplementary Section VB). The results of our analysis are depicted in Fig. 5 for the case of one and three DS. With three DS, we notice in the vicinity of $\mu=1$ the existence of not one but three quasi-degenerate Floquet multipliers. We analyzed the eigenvectors associated with these neutral modes and found that they correspond to relative translations of each DS, further confirming, beyond the observation of correlated or uncorrelated motion in Fig. 4r,d, their independence and defining mathematically the concept of temporal localization within the framework of delayed systems. Interestingly, the analysis of bound states yielded a single multiplier $\mu=1$ confirming that the ensemble moves as a single entity. Finally, the presence of many weakly damped oscillatory modes in Fig. 5 explains the variations of height and the sensitivity of the system to noise.

A description based on the Stokes parameters for polarized light allows interpreting the anti-phase dynamics as rotations along the equator of the Stoke's sphere, thus unveiling the vectorial character of the DS. We represent in Fig. 6a the temporal trace corresponding to one DS. It is found that the orbit proceeds essentially along the equator of the sphere $\left(\left|S_{3}\right| \lesssim 0.1\right)$. The system starts from the stable quasi-linearly polarized state represented by a green circle and performs a full clockwise rotation to reach the blue circle. Because these two polarizations are degenerate in a representation based solely upon the intensity dynamics of $\mathrm{X}$ and $\mathrm{Y}$, one would think having reached the initial point. Yet, it is only after receiving the second delayed perturbation after an additional time delay $\Delta \tau$ that the polarization cycle is closed. Incidentally, this explains why the secondary kink in Fig. 4a,b is upward since one must pass through the pure Y emission state $\left(S_{1}=-1\right)$ during this secondary plateau. Such dynamics along the equator is depicted in Fig. 6b where one can clearly identify two plateaus corresponding to the aforementioned symmetrical polarizations.

Such phase kinks are reminiscent of the Sine-Gordon phase equation which is known to give topological kinks and anti-kinks solutions. In our case, the phase must be interpreted as the orientation of the polarization. By applying a multiple time scale to our model (Supplementary Section V V as well as [38] for details) one reduces the dynamics to the sole orientation angle over the equatorial plane $\Phi$, such that $\left(S_{1}, S_{2}\right)=(\cos \Phi, \sin \Phi)$. The equation governing the dynamics of $\Phi$ reads

$$
\frac{\dot{\Phi}}{2}=\left(\alpha \gamma_{p}+\gamma_{a}\right) \sin \Phi+\bar{\eta} \sin \frac{\Phi^{\tau_{f}}}{2} \cos \frac{\Phi}{2}-\bar{\beta} \sin \frac{\Phi}{2} \sin \frac{\Phi^{\tau_{r}}}{2},
$$

with $\Phi^{\tau_{f, r}}=\Phi\left(t-\tau_{f, r}\right)$ the delayed arguments. This reduced model clarifies which parameters control the dynamics: the magnitude of the effective PSF and XPR rates dressed by the $\alpha$ factor, $(\bar{\eta}, \bar{\beta})=(\eta, \beta) \sqrt{1+\alpha^{2}}$, the dichroism $\gamma_{a}$ and the birefringence $\gamma_{p}$. The results of our reduced model is in quantitative agreement with the results of the full model and was indeed used to generate Fig. 6b. Elaborating upon these predictions, we reduced $\Delta \tau$ in order to obtain a single uninterrupted cycle and we were able to find DS as short at $25 \mathrm{ps}$, both in the full and the simplified model (Supplementary material Section VD. Also, exploiting the symmetry property $(\Phi, \beta) \rightarrow(-\Phi,-\beta)$, we deduce the existence of topological anti-kink solutions, which are depicted in Fig. 6. Notice that the kink and the anti-kink can not be separated in our measurements. More complicated kinks and anti-kinks that do not correspond to entire rotations also exist and will be the topic of further studies.

In summary, we demonstrated the existence of vectorial dissipative solitons and molecules in a VCSEL enclosed in a double external cavity. A simple theoretical model was found to reproduce the main experimental fea- 

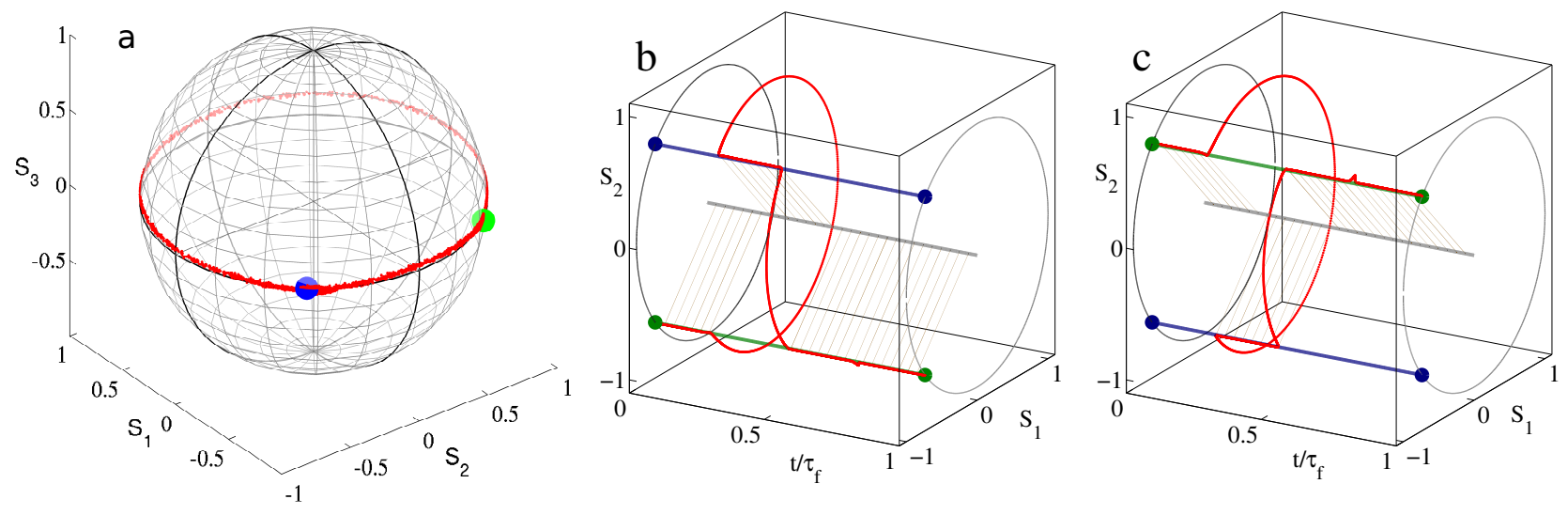

Figure 6. Temporal trace using the normalized Stokes representation of the dynamics. (a) Superposition of $10^{3}$ consecutive round-trips for a single soliton. One notices that the polarization angle performs a full cycle, but remains essentially close to the equatorial plane as indicated by the weakness of the $S_{3}$ component. The position of the stable fix point is denoted in green while the blue circle represents the symmetrical polarization with respect to the $\mathrm{Y}$ axis of symmetry . We recall that pure $\mathrm{X}$ and Y emission correspond to the equatorial point $S_{1}=1$ and $S_{1}=-1$, respectively, and that $\pm 45^{\circ}$ emission correspond to $S_{2}= \pm 1$. A single orbit unfolded in time is presented in (b). Here, one notices the existence of two plateaus corresponding to the anti-phase dip followed by the small inverted kink replica after a time $\Delta \tau$. A stable anti-kink was generated in (c) by inverting the phase of the XPR, i.e. setting $\beta \rightarrow-\beta$.

tures and the pulse shadow was identified as the pinning mechanism allowing the creation of molecules. The proof of independence was given experimentally by the study of the DS random motion and theoretically by the analysis of the Floquet multipliers. We interpreted the DS as rotations along the equatorial plane of the Stokes sphere and a multiple time-scale analysis allowed us to reduce the dynamics to a single delayed equation for the polarization orientation.

\section{ACKNOWLEDGEMENTS}

J.J. acknowledges financial support from the Ramón y Cajal fellowship and useful discussions with B. Krauskopf. J.J. and S.B. acknowledge financial support from project RANGER (TEC2012-38864-C03-01) and from the Direcció General de Recerca, Desenvolupament Tecnològic i Innovació de la Conselleria d'Innovació, Interior i Justícia del Govern de les Illes Balears co-funded by the European Union FEDER funds. M.G. acknowledge discussions with G. Giacomelli and C. Miniatura. The INLN group acknowledges funding of Région "ProvenceAlpes-Cote d'Azur" with the "Projet Volet Générale 2011 : Génération et Détection des Impulsions Ultra Rapides (GEDEPULSE)" and project ANR "OPTIROC" and project ANR - Jeunes Chercheurs " MOLOSSE ".

\section{Author Contributions}

M. Marconi performed the experimental characterization under the supervision of M. Giudici and assisted by S. Barland. M. Marconi, S. Balle and M. Giudici per- formed the statistical analysis of the experimental data. J. Javaloyes developed the theoretical model, the numerical analysis and wrote the manuscript together with S. Balle and M. Giudici. All the authors participated to the interpretation of the results.

\section{Competing Interests}

The authors declare that they have no competing financial interests.

\section{Materials \& Correspondence}

Correspondence and requests for materials should be addressed to Massimo Giudici (email: massimo.giudici@inln.cnrs.fr).

\section{METHODS}

\section{A. Setup and VCSEL details}

Two different lasers from ULM-photonics lasing at 850 nm (ULM.850-PMTNS46FOP) have been used. They are single transversal mode lasers with a suppression ratio larger than $10 \mathrm{~dB}$ at the rated power of $1 \mathrm{~mW}$. Both emit linearly polarized light and exhibit a birefringence around $7.5 \mathrm{GHz}$. Their low dichroism and birefringence helps to maximize the effects of PSF and XPR. Their threshold is around $0.5 \mathrm{~mA}$ with the bluest polarization mode, that we call Y, appearing at threshold. Both devices exhibit a small dichroism and polarization switching 
can be implemented by injecting an external linearly polarized beam of low power $(<100 \mathrm{nW})$. It is important to underline that the linear polarization component selected by the PSF cavity and used for XPR must be the dominant component appearing at threshold of the solitary laser which is Y, the bluest one, in our case. The DS are obtained by choosing some specific values of the system parameters. Typical feedback rate for this regime is around $0.5 \%$, while the XPR rate is about $1-2 \%$. Pumping currents are $2.6 \mathrm{~mA}<J<3.1 \mathrm{~mA}$. In this experimental analysis we explored $\tau_{f}$ values ranging from $1.3 \mathrm{~ns}$ up to $10 \mathrm{~ns}$. The first value is the lowest limit possible with our setup, while the second one is just the maximum value explored. The detection set-up consists of two $8 \mathrm{GHz}$ detectors combined with a $33 \mathrm{GHz}$ scope (Tektronix DPO73304D).

\section{B. Theoretical model}

In order to theoretically analyze the behavior of the VCSEL and compare with the experimental results, we use the Spin-Flip Model (SFM) [43], suitably modified for incorporating the effects of both polarization selective feedback and crossed-polarization re-injection. We adopt a mixed description in terms of linearly polarized components of the field, $\mathrm{X}$ and Y, where PSF and crossed-polarization re-injection are easily expressed, and circularly polarized components of the field, $E_{ \pm}=$ $(X \pm i Y) / \sqrt{2}$, where the SFM is naturally expressed. In this framework, the dynamics of the system is written as

$$
\begin{aligned}
\dot{X} & =(1+i \alpha)\left[\frac{G_{+} E_{+}+G_{-} E_{-}}{\sqrt{2}}-X\right]-z X \\
& +\beta e^{-i a} Y\left(t-\tau_{r}\right) \\
\dot{Y} & =(1+i \alpha)\left[\frac{G_{+} E_{+}-G_{-} E_{-}}{i \sqrt{2}}-Y\right]+z Y \\
& +\eta e^{-i \Omega} Y\left(t-\tau_{f}\right) \\
T \dot{D}_{ \pm} & =\mu-D_{ \pm}-G_{ \pm}\left|E_{ \pm}\right|^{2} \mp \frac{\gamma_{J}}{\gamma_{e}}\left(D_{+}-D_{-}\right)
\end{aligned}
$$

where $\alpha$ is Henry's linewidth enhancement factor, $D_{ \pm}$ are the scaled carrier densities in each spin channel and $G_{ \pm}=D_{ \pm}\left(1-\varepsilon\left|E_{ \pm}\right|^{2}\right)$ is the gain for each circularly polarized field component including gain saturation. The terms $Y\left(t-\tau_{f}\right)$ and $Y\left(t-\tau_{r}\right)$ in the evolution equations for the $\mathrm{X}$ and $\mathrm{Y}$-components describe the effects of PSF and of XPR, which have strengths $\eta$ and $\beta$, phases $\Omega$ and $a$, and time delays $\tau_{f}$ and $\tau_{r}$, respectively.

In equations (1,3), time has been scaled to the cavity decay rate $\kappa, T=\kappa / \gamma_{e}$ represents the scaled carrier lifetime and $\gamma_{J}$ is the decay rate of the spin-difference. The scaled density of carriers injected per unit time into the active region due to the bias current normalized to threshold is $\mu$. In addition, we defined $z=\left(\gamma_{a}+i \gamma_{p}\right) / \kappa$ where $\gamma_{a}$ (resp. $\gamma_{p}$ ) describes the linear dichroism (resp. birefringence) of the cavity. Finally, we have added to the time evolution in Eqs. (1,3) independent Langevin sources describing noise due to spontaneous emission and current fluctuations [45] with variance $\xi \sim 10^{-3}$. We assume typical values $\alpha=2, \varepsilon_{g}=2 \times 10^{-2}, \gamma_{a}=0, \gamma_{p}=$ $5 \times 10^{-2}, \mu=10, T=500$ and $\gamma_{J}=60 \gamma_{e}$ which - taking $\kappa^{-1}=2 \mathrm{ps}$ - correspond to a carrier lifetime $\gamma_{e}^{-1}=1 \mathrm{~ns}$, a spin-difference decay time of $\gamma_{J}^{-1}=16.6 \mathrm{ps}$, a frequency splitting $\gamma_{p} \kappa / \pi \sim 8 \mathrm{GHz}$ and a relaxation oscillation frequency $\nu_{r}=(2 \pi)^{-1} \sqrt{2(\mu-1) \gamma_{e} \kappa} \sim 15 \mathrm{GHz}$. The values of the PSF and XPR parameters used in all theoretical figures are $\eta=0.09$ and $\beta=0.06$ while $\Omega=a=0$. The time delays in Fig. 4 a,b, Fig. 5 and Fig. 6 are $\tau_{f}=1500$ and $\tau_{r}=1600$ and correspond to $3 \mathrm{~ns}$ and $3.2 \mathrm{~ns}$. We took $\tau_{r}=1700$ (i.e. $3.4 \mathrm{~ns}$ ) in Fig. 4 4 ,d in order to better visualize the shadow of the pulse and $\tau_{r}=1900$ (i.e. $3.6 \mathrm{~ns}$ ) in Fig. 6b,c in order to clarify the existence of the plateaus. For completeness, we recall that the normalized Stokes parameters read

$$
\begin{gathered}
S_{0}=|X|^{2}+|Y|^{2}, S_{1}=\left(|X|^{2}-|Y|^{2}\right) / S_{0} \\
S_{2}=2 \Re\left(X Y^{\star}\right) / S_{0}, S_{3}=-2 \Im\left(X Y^{\star}\right) / S_{0} .
\end{gathered}
$$

\section{Numerical simulations}

Eqs. 133 were numerically integrated with a fourthorder Runge-Kutta method with constant step size $(h=$ $10^{-2}$ ) 46]. The delayed contributions in Eqs. (12 2 demand a special care. To advance the solution with a step $h$ from $t_{n}=n h$ to $t_{n+1}$, the Runge-Kutta algorithm requires the values of $Y\left(t-\tau_{f, r}\right)$ at intermediate points $t_{n+1 / 2}$. These are not known and must be interpolated from past values with an order of approximation consistent with that of the algorithm of integration. Therefore, besides keeping memory of the past values of $Y$ we also retain the past values of the time derivative $\dot{Y}(t)$. Such a method allows building a third order Hermite polynomial approximation for $Y(t)$ between the time $\left(t_{n}-\tau_{f, r}\right)$ and $\left(t_{n+1}-\tau_{f, r}\right)$. By evaluating this interpolating polynomial at $\left(t_{n+1 / 2}-\tau_{f, r}\right)$, we ensure an overall fourth order accuracy. Finally, the stochastic noise contribution in Eqs. (1-3) is added after the deterministic step by simply using the Euler method [46] and scaling $\xi$ by $\sqrt{h}$.

Solitons can be generated numerically by starting from a random initial condition around the off solution; during the transient regime, several spikes corresponding to the so-called relaxation oscillations are generated which can result in one or several DS in the asymptotic regime. A more controlled approach is however more appropriate to generate molecules. We use as an initial condition the CW mode, i.e. the solution with 0 -DS . From this state, by inverting the feedback phase $\Omega \rightarrow \Omega+\pi$ one can generate polarization slips that will eventually stabilize as vectorial DS after a few tens round-trips in the cavity. Notice that setting the phase back to its original value, even after a short time interval generates another Soliton and if the interval is too short no DS is generated. From this newly found periodic solution, additional perturbations can be applied in order to generate multiple 
independent solitons or soliton molecules if the perturba-

tion is properly timed.

[1] N. Akhmediev and A. Ankiewicz. Dissipative Solitons, volume 661 of Lecture Notes in Physics. Springer Berlin Heidelberg, 2005.

[2] N. Akhmediev and A. Ankiewicz. Dissipative Solitons: From Optics to Biology and Medicine Series, volume 751 of Lecture Notes in Physics. Springer Berlin Heidelberg, 2008.

[3] T. Ackemann, W. J. Firth, and G.L. Oppo. Chapter 6 fundamentals and applications of spatial dissipative solitons in photonic devices. In P. R. Berman E. Arimondo and C. C. Lin, editors, Advances in Atomic Molecular and Optical Physics, volume 57 of Advances In Atomic, Molecular, and Optical Physics, pages 323 - 421. Academic Press, 2009.

[4] O. Descalzi, M. Clerc, S. Residori, and G. Assanto. Localized States in Physics: Solitons and Patterns, volume 751 of Lecture Notes in Physics. Springer Berlin Heidelberg, 2011.

[5] P. Grelu and N. Akhmediev. Dissipative solitons for mode-locked lasers. Nat Photon, 6(2):84-92, 2012.

[6] G. Nicolis and I. Prigogine. Self-Organization in Nonequilibrium Systems: From Dissipative Structures to Order through Fluctuations. Wiley, 1977.

[7] P. Coullet, C. Riera, and C. Tresser. A new approach to data storage using localized structures. Chaos, 14:193201, Mar 2004.

[8] S. Barland, J. R. Tredicce, M. Brambilla, L. A. Lugiato, S. Balle, M. Giudici, T. Maggipinto, L. Spinelli, G. Tissoni, T. Knodl, M. Miller, and R. Jager. Cavity solitons as pixels in semiconductor microcavities. Nature, 419(6908):699-702, Oct 2002.

[9] F. Leo, S. Coen, P. Kockaert, S.P. Gorza, P. Emplit, and M. Haelterman. Temporal cavity solitons in onedimensional kerr media as bits in an all-optical buffer. Nat Photon, 4(7):471-476, Jul 2010.

[10] T. Elsass, K. Gauthron, G. Beaudoin, I. Sagnes, R. Kuszelewicz, and S. Barbay. Fast manipulation of laser localized structures in a monolithic vertical cavity with saturable absorber. Applied Physics B, 98(2-3):327331, 2010.

[11] P. Genevet, S. Barland, M. Giudici, and J. R. Tredicce. Cavity soliton laser based on mutually coupled semiconductor microresonators. Phys. Rev. Lett., 101:123905, Sep 2008.

[12] S. Fauve and O. Thual. Solitary waves generated by subcritical instabilities in dissipative systems. Phys. Rev. Lett., 64:282-284, Jan 1990.

[13] W. Chang, J. M. Soto-Crespo, A. Ankiewicz, and N. Akhmediev. Dissipative soliton resonances in the anomalous dispersion regime. Phys. Rev. A, 79:033840, Mar 2009.

[14] N. N. Rosanov and G. V. Khodova. Autosolitons in bistable interferometers. Opt. Spectrosc., 65:449, 1988.

[15] M. Tlidi, P. Mandel, and R. Lefever. Localized structures and localized patterns in optical bistability. Phys. Rev. Lett., 73:640-643, Aug 1994.

[16] W. J. Firth and A. J. Scroggie. Optical bullet holes: Robust controllable localized states of a nonlinear cavity.
Phys. Rev. Lett., 76:1623-1626, Mar 1996.

[17] L.A. Lugiato. Introduction to the feature section on cavity solitons: An overview. Quantum Electronics, IEEE Journal of, 39(2):193-196, 2003.

[18] P. Coullet, C. Riera, and C. Tresser. Stable static localized structures in one dimension. Phys. Rev. Lett., 84:3069-3072, Apr 2000.

[19] D. Y. Tang, W. S. Man, H. Y. Tam, and P. D. Drummond. Observation of bound states of solitons in a passively mode-locked fiber laser. Phys. Rev. A, 64:033814, Aug 2001.

[20] M. Stratmann, T. Pagel, and F. Mitschke. Experimental observation of temporal soliton molecules. Phys. Rev. Lett., 95:143902, Sep 2005.

[21] P. Grelu and J.M. Soto-Crespo. Temporal soliton "molecules" in mode-locked lasers: Collisions, pulsations, and vibrations. In Dissipative Solitons: From Optics to Biology and Medicine, volume 751 of Lecture Notes in Physics, pages 1-37. Springer Berlin Heidelberg, 2008.

[22] D. Turaev, A. G. Vladimirov, and S. Zelik. Long-range interaction and synchronization of oscillating dissipative solitons. Phys. Rev. Lett., 108:263906, Jun 2012.

[23] J. K. Jang, M. Erkintalo, S. G. Murdoch, and S. Coen. Ultraweak long-range interactions of solitons observed over astronomical distances. Nat Photon, 7(8):657-663, Aug 2013. Article.

[24] S. Chouli and P. Grelu. Soliton rains in a fiber laser: An experimental study. Phys. Rev. A, 81:063829, Jun 2010.

[25] S. T. Cundiff, J. M. Soto-Crespo, and N. Akhmediev. Experimental evidence for soliton explosions. Phys. Rev. Lett., 88:073903, Feb 2002.

[26] J. U. Kang, G. I. Stegeman, J. S. Aitchison, and N. Akhmediev. Observation of manakov spatial solitons in AlGaAs planar waveguides. Phys. Rev. Lett., 76:3699 3702, May 1996.

[27] Q. L. Williams, J. García-Ojalvo, and R. Roy. Fast intracavity polarization dynamics of an erbium-doped fiber ring laser: Inclusion of stochastic effects. Phys. Rev. A, 55:2376-2386, Mar 1997.

[28] H. Zhang, D. Y. Tang, L. M. Zhao, and X. Wu. Observation of polarization domain wall solitons in weakly birefringent cavity fiber lasers. Phys. Rev. B, 80:052302, Aug 2009.

[29] C. Lecaplain, P. Grelu, and S. Wabnitz. Polarizationdomain-wall complexes in fiber lasers. J. Opt. Soc. Am. $B, 30(1): 211-218$, Jan 2013.

[30] K. D. Choquette, D. A. Richie, and R. E. Leibenguth. Temperature dependence of gain-guided vertical-cavity surface emitting laser polarization. Applied Physics Letters, 64(16):2062-2064, 1994.

[31] K.D. Choquette, R.P. Schneider, K.L. Lear, and R.E. Leibenguth. Gain-dependent polarization properties of vertical-cavity lasers. Selected Topics in Quantum Electronics, IEEE Journal of, 1(2):661 -666, jun 1995.

[32] F. Marino, L. Furfaro, and S. Balle. Cross-gain modulation in broad-area vertical-cavity semiconductor optical amplifier. Applied Physics Letters, 86(15):151116, 2005.

[33] M. Virte, K. Panajotov, H. Thienpont, and M. Scia- 
manna. Deterministic polarization chaos from a laser diode. Nat Photon, 7(1):60-65, 2013.

[34] G. Giacomelli and A. Politi. Relationship between delayed and spatially extended dynamical systems. Phys. Rev. Lett., 76:2686-2689, Apr 1996.

[35] J. Javaloyes and S. Balle. Multimode dynamics in bidirectional laser cavities by folding space into time delay. Opt. Express, 20(8):8496-8502, Apr 2012.

[36] A. Perez-Serrano, J. Javaloyes, and S. Balle. Spectral delay algebraic equation approach to broad area laser diodes. Selected Topics in Quantum Electronics, IEEE Journal of, $\operatorname{PP}(99): 1-1,2013$.

[37] M. Marconi, J. Javaloyes, S. Barland, M. Giudici, and S. Balle. Robust square-wave polarization switching in vertical-cavity surface-emitting lasers. Phys. Rev. A, 87:013827, Jan 2013.

[38] J. Javaloyes, M. Marconi, and M. Giudici. Phases dynamics in VCSELs with delayed optical feedback and cross re-injection. Phys. Rev. A, not submitted, 2014, http://arxiv.org/abs/1405.7493.

[39] J. Javaloyes, J. Mulet, and S. Balle. Passive mode locking of lasers by crossed-polarization gain modulation. Phys. Rev. Lett., 97:163902, Oct 2006.

[40] F. T. Arecchi, G. Giacomelli, A. Lapucci, and R. Meucci. Two-dimensional representation of a delayed dynamical system. Phys. Rev. A, 45:R4225-R4228, Apr 1992.

[41] H. U. Bödeker, M. C. Röttger, A. W. Liehr, T. D. Frank, R. Friedrich, and H.-G. Purwins. Noise-covered drift bifurcation of dissipative solitons in a planar gas-discharge system. Phys. Rev. E, 67:056220, May 2003.

[42] Yaroslav V. Kartashov, Victor A. Vysloukh, and Lluis Torner. Brownian soliton motion. Phys. Rev. A, 77:051802, May 2008.

[43] M. San Miguel, Q. Feng, and J. V. Moloney. Lightpolarization dynamics in surface-emitting semiconductor lasers. Physical Review A (Atomic, Molecular, and Optical Physics), 52(2):1728-1739, 1995.

[44] B. Schäpers, M. Feldmann, T. Ackemann, and W. Lange. Interaction of localized structures in an optical patternforming system. Phys. Rev. Lett., 85:748-751, Jul 2000.

[45] J. Mulet, C. R. Mirasso, and M. San Miguel. Polarization resolved intensity noise in vertical-cavity surface-emitting lasers. Physical Review A (Atomic, Molecular, and Optical Physics), 64(023817), 2001.

[46] William H. Press, Saul A. Teukolsky, William T. Vetterling, and Brian P. Flannery. Numerical Recipes: The Art of Scientific Computing. Cambridge University Press, August 2007.

[47] R. Lehoucq and D. Sorensen. Deflation techniques for an implicitly restarted arnoldi iteration. SIAM Journal on Matrix Analysis and Applications, 17(4):789-821, 1996.

[48] Koen Engelborghs, Tatyana Luzyanina, and Giovanni Samaey. Dde-biftool v. 2.00: a matlab package for bifurcation analysis of delay differential equations. Technical report, Department of Computer Science, K.U.Leuven, Belgium., 2001.
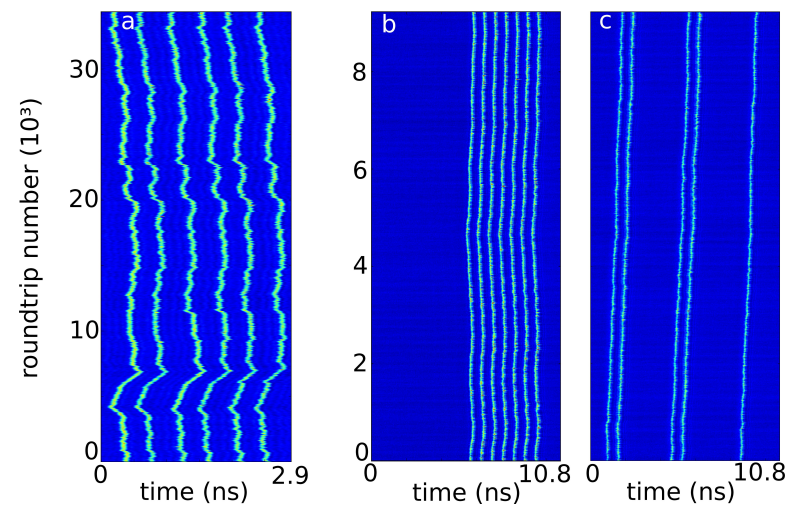

Figure 7. Panel a): Space-time like diagram of a 6-DS molecule obtained for the same parameters of Fig. 3 a $\left(\tau_{f}=\right.$ $2.9 \mathrm{~ns}$ and $\left.\tau_{r}=3.3 \mathrm{~ns}\right)$. By increasing $\tau_{f}$, a larger number of independent DS and molecules with a higher number of elements can fit into the cavity. Panel b): Space-time like diagram of a molecule of 7-DS evolving in a limited portion of an external cavity having $\tau_{f}=10.8 \mathrm{~ns}$ and $\tau_{r}=11.1 \mathrm{~ns}$. Panel c): Another situation obtained for the same parameter as panel b) showing three independent DS: a single DS and two 2-DS molecules.

\section{SUPPLEMENTARY MATERIAL}

\section{A. Complexes and bond distances}

In the parameter region where vectorial DS exist multiple situations with different number of independent DS and/or DS molecules evolving within the external cavity are observed for the same values of the parameters. This multiplicity is a consequence of the multi-stability between each DS and the CW solution. In Fig. 3a-c we have shown three of those situations for $\tau_{f}=2.9 \mathrm{~ns}$ and $\tau_{r}=3.3 \mathrm{~ns}$. In Fig. $7 \mathrm{a}$, we depict for the same parameters a situation where six DS are bound together to form a molecule of six elements. The molecule exhibits a random walking as a function of the round-trip covered, wandering inside the cavity, but the distance between its elements is maintained. In Fig. 8, we analyze statistically the evolution of the 3-DS molecule of Fig. $3 \mathrm{p}$ and the 6-DS molecule shown in Fig. $7 \mathrm{a}$, as well as their bond distances. Panels a and $\mathrm{c}$ of Fig. 8 display the probability distribution of the position of the first peak of respectively the 3-DS molecule and of the 6-DS molecule. This curve has been obtained analyzing the evolution of the molecules on $36 \times 10^{3}$ round-trips. Both panels indicate that the molecules wander inside the external cavity in a range of approximately $400 \mathrm{ps}$ ( $300 \mathrm{ps}$ for the 6 -DS molecules) and, in this visited range, the probabilities of the positions are quite uniform, thus indicating that there are no preferential positions. The probability distribution of the bond distances (panel b) for the 3-DS molecule and panel d) for the 6-DS molecule reveals that the separation between solitons in a molecule is either maintained during the evolution or jumps between well 
defined values. In the case of the 3-DS molecule the bond distance is centered at $480 \mathrm{ps}$ both for the first to the second soliton and for the second to the third. This bond distance corresponds to $\tau_{r}-\tau_{f}$, as explained by the theoretical model. Fluctuations of this bond distance during the molecule evolution are at the limit of the sampling resolution of the scope (the standard deviation is approximately $10 \mathrm{ps}$ ).

In the case of the 6 -DS molecule the bond distances are peaked to different values depending on the couple of peaks considered. The existence of discrete bond distances is a common feature for dissipative solitons and it was found already in the case of spatial DS [44]. Three values are observed considering all the neighbor distances: $380 \mathrm{ps}, 500 \mathrm{ps}$ and $600 \mathrm{ps}$ (for clarity in panel d) only the distances between two couples of elements are considered). For some couple of elements (for example the second and the third peak), the bond distance can change during the evolution and, in this case, it may jump to another value. Jumps observed are of $120 \mathrm{ps}$, and the possible bond distances observed correspond to one of the three values described before. It is worth remembering that $120 \mathrm{ps}$ is the inverse of the birefringence of the laser used. This suggests that, while the main bond distances are fixed by $\Delta \tau=\tau_{r}-\tau_{f}$, multiples, discrete sub-distances exist and are ruled by birefringence. This feature has been also found in our theoretical analysis.

When the size of the external cavity is increased, larger molecules and a larger number of independent DS can be hosted in the external cavity. As examples, in Fig. $7 \mathrm{~b}, \mathrm{c}$, we show two situations obtained for a value of $\tau_{f}=10.8 \mathrm{~ns}$ and $\tau_{r}=11.1 \mathrm{~ns}$. In panel b) we show a 7 DS molecule which occupies a limited size of the cavity. The bond distance is given by $2 \Delta \tau=600 \mathrm{ps}$. In panel c) we show three independent DS: two 2-DS molecules, both having a bond distance of $600 \mathrm{ps}$, and a single DS.

\section{B. Floquet analysis}

Eqs. (1.3) have different periodic solutions, and their linear stability can be determined via a Floquet analysis, which implies the reconstruction of the monodromy operator $\mathcal{M}$ for the perturbations to the periodic solutions. A periodic solution is stable if the whole spectrum of the associated Floquet multipliers is composed of complex numbers having modulus less than one, and unstable otherwise. A Floquet multiplier equal to one corresponds to a neutral mode, and it is typically associated with an invariance of the system, e.g., translational invariance of a solution. If a periodic solution consists of $N$ pulses that can be freely displaced one relatively to the other within the period, $N$ Floquet multipliers equal to 1 must exist. In the case of $N$ independent DS, small deviations from unity for these multipliers may appear due to the residual interactions for finite values of the time delay $\tau_{f}$.

In our case, the operator $\mathcal{M}$, although a priori infinite dimensional, reduces to a square matrix of size $\tau / \delta t$ due to the discrete sampling incurred by the constant stepsize numerical algorithm. It is constructed by taking one arbitrary point of the periodic orbit and inserting a small perturbation in all the degrees of freedom as represented by the mesh points in the delay time and let the system evolve over an entire period. The deviation of the end point from the unperturbed orbit yields a column of the operator $\mathcal{M}$. The eigenvalues (i.e. the Floquet's multipliers) and eigenvectors of $\mathcal{M}$ can be calculated with a complete decomposition method (i.e. the QR method [46]), although this is not efficient for large sizes of $\mathcal{M}$. In our case, $\operatorname{dim}(\mathcal{M}) \sim 10^{5}$ or higher, and it is more efficient to use the Implicitly Restarted Arnoldi Method 47]. Although this latter method provides only the dominant eigenvalues (i. e., those with the largest modulus), it allows us to assess the stability of the periodic solutions and to compute the dominant Floquet multipliers. We verified that both methods give identical results.

The results were found also to be in agreement with the one given by DDEBIFTool 48 for the orbits with one and two DS. However, it was not possible to use such software in general since we do not have a bifurcation scenario for the apparition of the DS. As such, we needed to jump-start the Newton process of convergence to the periodic solution from a guess orbit found via direct numerical integration. Consequently, we faced the problem of the weak convergence of the Newton method in a high dimensional space. While correcting orbits with one or two DS was possible, this process became increasingly difficult for larger numbers of DS. This is due to the fact that in each case the adaptive mesh discretization had to be increased to accommodate for the larger number of localized temporal structures increasing the dimensionality of the problem.

\section{Vectorial phase model}

We mention briefly the derivation of the phase model that can be found in 38. Far from threshold, the fluctuations of the total intensity die out rapidly and the dynamics remains confined on a Stokes sphere of a given radius. In addition, strongly elliptical states would incur a large energetic penalty due to the imbalance between the two carrier reservoirs. This further confines the residual dynamics to the vicinity of the equatorial plane of the Stokes sphere. Without external perturbation, one may not expect any complex residual dynamics in such situations from Eq. (1,3) since the center manifold is only two dimensional. Besides, the center manifold consists in two decoupled variables, the polarization orientation angle $\Phi$ and the optical phase.

Notwithstanding, the coherent delayed retro-actions imposed by the feedback terms in Eqs. (12 make it so that the optical phase of the field couples back into the dynamics. As such our reduced model will consist in a "vectorial" phase for the orientation of the quasi-linear polarization as well as for the optical phase of the field. 

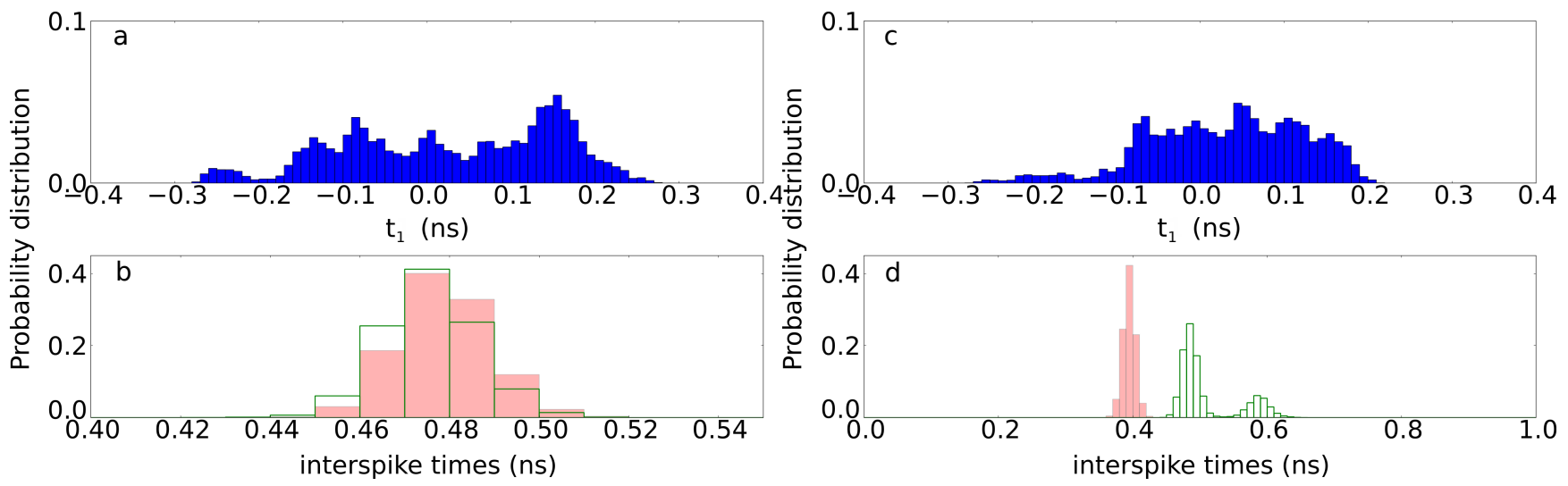

Figure 8. Panel a: Probability distribution of the position of the first DS within the cavity $\left(\Delta_{n}\right)$ for the space-time like diagram plotted in Fig. 3b. Panel b: Probability distribution function for the separation between the second and the first DS (pink trace) and between the third and the second DS (green trace) of the molecule of Fig. 3b. Panel c: Same as in a) but for the first DS of the space-time like diagram plotted in Fig. $7 \mathrm{f}$. Panel d: Probability distribution function for the separation between the second and the first DS (pink trace) and between the third and the second DS (green trace) of the 6-DS molecule of Fig. 7 a.

After applying a multiple time scale analysis to Eq. 1. 3) considering the relaxation oscillation as a smallness parameter (see [38] for more details), we obtain a phase model for the orientation, i.e. the difference between the two circular components of the field $\psi_{ \pm}, \Phi=\psi_{+}-\psi_{-}$and the global optical phase represented conveniently by the half sum $\Sigma=\left(\psi_{+}+\psi_{-}\right) / 2$. The reduced model with $\mathrm{PSF}$ and XPR, which is valid only far from threshold, reads

$$
\begin{aligned}
& \dot{\Sigma}=\left(\alpha \gamma_{a}-\gamma_{p}\right) \cos \Phi-\bar{\eta} \sin \frac{\Phi^{\tau_{f}}}{2} \sin \frac{\Phi}{2} \sin \left(u+\Omega+\Sigma-\Sigma^{\tau_{f}}\right)-\bar{\beta} \cos \frac{\Phi}{2} \sin \frac{\Phi^{\tau_{r}}}{2} \sin \left(u+a+\Sigma-\Sigma^{\tau_{r}}\right) \\
& \frac{\dot{\Phi}}{2}=\left(\gamma_{a}+\alpha \gamma_{p}\right) \sin \Phi+\bar{\eta} \sin \frac{\Phi^{\tau_{f}}}{2} \cos \frac{\Phi}{2} \cos \left(u+\Omega+\Sigma-\Sigma^{\tau_{f}}\right)-\bar{\beta} \sin \frac{\Phi}{2} \sin \frac{\Phi^{\tau_{r}}}{2} \cos \left(u+a+\Sigma-\Sigma^{\tau_{r}}\right)
\end{aligned}
$$

with $(\bar{\eta}, \bar{\beta})=(\bar{\eta}, \bar{\beta}) \sqrt{1+\alpha^{2}}$ and $u=\arctan \alpha$. It is worthwhile to notice that these two phases $\Phi$ and $\Sigma$ are of very different nature: while the optical phase precise value $\Sigma$ is irrelevant due to the phase invariance in an autonomous system the orientation phase $\Phi$ value is a meaningful quantity. This is due to the broken rotational invariance imposed by the dichroism and the birefringence of the VCSEL cavity.

In the case of a monochromatic solution, the orientation of the quasi linear polarization $\Phi$ reaches a fix point while the half sum $\Sigma \sim \omega t$ drifts at the frequency of the mode under consideration. The modal structure of Eqs. 45 is a complex problem and is reported in 38 . In the case of the DS presented in this manuscript, hundreds of such monochromatic solutions defined by doublets $[\omega, \Phi(\omega)]$ exist. The analysis of such modes is useful for explaining the DS in the sense that we identified that some modes verify the resonance condition $u+\Omega+\omega \tau_{f}=n \pi$ and $u+a+\omega \tau_{r}=m \pi$. In this case, by writing $\Sigma=\omega t+\delta$ with $\delta \ll 1$, one can decouple the fluctuations of the optical phase $\delta$ dynamics from the orientation dynamics and reduce the dynamics to a single equation for $\Phi$.

\section{Multiple binding distances and fast dissipative solitons orbits}

From the analysis presented in Figs. 46 one understands that the DS is composed of two plateaus for the polarization orientation. The length of the second plateau is controlled by the difference between the two delays $\Delta \tau$. One can appreciate in Fig. 9 a-c that because the replica of the main anti-phase dip is re-injected after a time $\Delta \tau$, the same is also true for this replica that is re-injected at time $2 \Delta \tau$, etc. This effect induces discrete binding distances that allows for the existence of molecules of different types. We present in in Fig. 9a-c, molecules with binding distance $\Delta \tau, 2 \Delta \tau$ and $4 \Delta \tau$. Obviously, the larger the distance, the smaller the replica and the weaker the binding force.

However, with the perspective of applications in mind, such multiple binding distances can be a hindrance and this second plateau can be reduced as much as the time needed to perform the first part of the orbit. Here, the secondary kink would be located at a very close distance, thereby negating this multiplicity of binding distances. This allows to close the polarization loop in Fig. 6 d-f by 

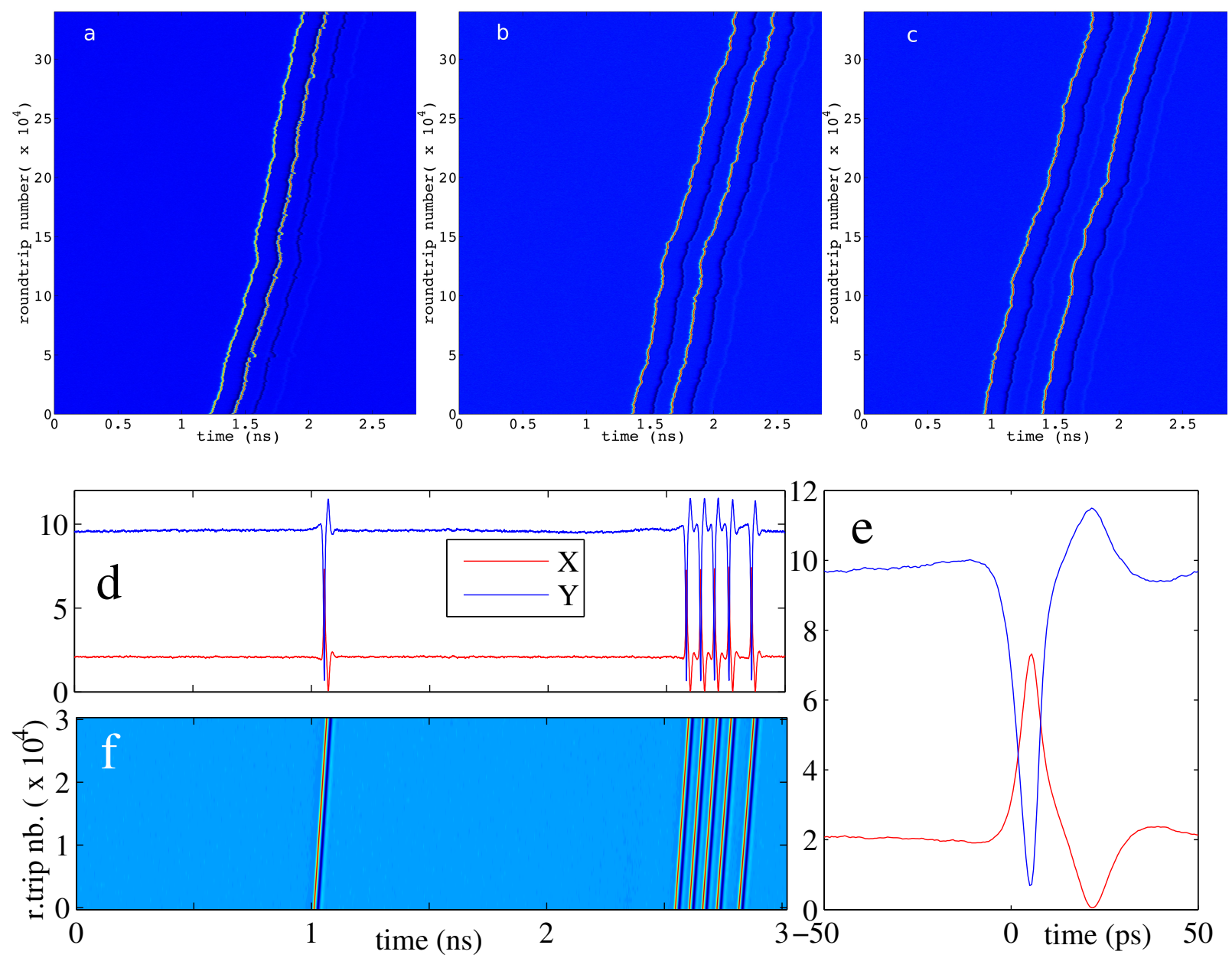

Figure 9. Several stable equilibrium distances are found and correspond to multiples of $\Delta \tau$ as represented in panels a,b, and c. The larger the distance, the weaker the binding force as indicated by the larger amount of fluctuations between the two elements of the molecule. For small values of $\Delta \tau$, the DS temporal extent can be reduced as depicted in the time trace in d) with $\gamma_{p}=0.1$ (corresponding to a frequency splitting of $15 \mathrm{GHz}$ ), PSF and XPR rates of $\eta=0.4$ and $\beta=0.26$, and time delays $\tau_{f}=3 \mathrm{~ns}$ and $\tau_{r}=\tau_{f}+18 \mathrm{ps}$. The full orbit considering the oscillatory tail represented in d) is around $25 \mathrm{ps}$. A complex bit sequence is represented in $\mathrm{f}$ ) over many round-trips signaling the stability of the setup to store information as polarization encoded bit sequences.

performing a single, uninterrupted, orbit. For larger values of the birefringence and of the PSF and XPR rates we obtain the results described in Fig. 9 $\mathrm{d}-\mathrm{f}$, which correspond to DS whose duration is $\sim 25 \mathrm{ps}$. Here only considering the FWHM would yield a much shorter width of $10 \mathrm{ps}$ although such short pulse-width should not be understood as an inverse effective bit rate. We estimate such bit-rate to be between 20 and $40 \mathrm{GHz}$. For instance, we store in Fig. $9 \mathrm{~d}$-f a bit pattern composed of several DS in order to demonstrate the robustness of the dynamics even in the presence of noise. Because the dynamics consists in a pure anti-phase, the carrier lifetime cannot be identified as the time-scale limiting the pulse-width. In addition, since the phase dynamics proceeds along the equator of the Stokes sphere or, equivalently, because the polarization remains always linear, there is no imbalance between the two population reservoirs indicating that the spin-flip time scale could also not be the ultimate limiting factor. Finally, because the PSF and the XPR rates govern the temporal extent of the DS, we think that superior results could even be achieved by using a VCSOA in an external double cavity instead of using a VCSEL. 